\title{
Non-Equilibrium Plasma Instabilities
}

\author{
J.P. Petit and J. Geffray \\ LAMBDA Laboratory, France*
}

\begin{abstract}
Low magnetic Reynolds non-thermal plasmas are sensitive to the Velikhov electrothermal instability when the Hall parameter exceeds the local critical value. Then the instability quickly grows and highly reduces the performance of MHD converters. After operating (International MHD Meeting, Warsaw 1968) the first high power density two-temperature - but short duration - MHD Faraday generator, using a first stabilization method, we presented a better one in the beginning of the eighties that was demonstrated through low pressure experiments (French Academy of Sciences, 1981; and 8th International MHD meeting, Moscow 1983). This second method was based on a non-uniform $B$-field and electrical tensor conductivity. It is time now to rebuild MHD activities all over the world as deserved, corresponding to progress of today's techniques. Different applications of this instability cancellation are presented.
\end{abstract}

PACS numbers: 52.30.Cv, 52.35.-g, 52.55.-s

\section{Introduction}

At the beginning of the sixties, many countries built MHD power plants, whose goal was to achieve direct conversion of thermal energy into electricity with a better efficiency than classical steam systems. In so-called open cycles, fossil fuels seeded with cesium were used. But the resistance limit of walls and electrodes was not sufficient to stand the thermal effects due to the high temperature required for a good electrical conductivity. Then closed cycles systems appeared, using noble gases (He) with Cs seed. In suitable conditions, non-thermal ionization could be achieved, with subsequent rise of the electrical conductivity. But in 1962 Velikhov predicted by calculation the disastrous effect of an electrothermal instability, also called the Velikhov instability [1] occurring in $T_{\mathrm{e}}>T_{\mathrm{g}}$ regimes, when the Hall parameter was higher than a critical value $R_{\mathrm{cr}}$. Let us call $\mu$ the electron mobility, $E_{\mathrm{i}}$ - the ionization energy, $n_{\mathrm{e}}$ - the electronic density, $\sigma$ - the scalar electrical conductivity and $E^{*}$ - the electric field. We get [2]:

$$
\begin{aligned}
& f=-\left(\frac{\delta \mu}{\mu}\right) /\left(\frac{\delta n_{\mathrm{e}}}{n_{\mathrm{e}}}\right), \\
& s=\frac{2 k T_{\mathrm{e}}^{2}}{E_{\mathrm{i}}\left(T_{\mathrm{e}}-T_{\mathrm{g}}\right)} \times \frac{1}{1+\frac{3}{2} \frac{k T_{\mathrm{e}}}{E_{\mathrm{i}}}}, \\
& \beta_{\mathrm{cr}}=1.935 f+0.065+s .
\end{aligned}
$$

Growth rate of the instability

$$
g=\frac{\sigma E^{* 2}}{n_{\mathrm{e}}\left(E_{\mathrm{i}}+\frac{3}{2} k T_{\mathrm{e}}\right)\left(1+\beta^{2}\right)}\left(\beta-\beta_{\mathrm{cr}}\right)
$$

weakly ionized gas

$$
\beta_{\mathrm{cr}} \cong\left(s^{2}+2 s\right)^{1 / 2},
$$

fully ionized gas

$$
\beta_{\mathrm{cr}} \cong 2+s \text {. }
$$

In Fig. 1, the typical distortion pattern of the electric current lines, due to the fast growing Velikhov instability (linear MHD channel) is shown.

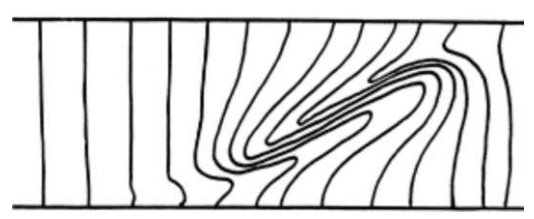

Fig. 1. Current line distortion in a Faraday converter due to electrothermal instability.

This strong electron density turbulence completely destroyed the performances of MHD generators, so that these projects, involving 2000 researchers in the world, were one after the other abandoned.

\section{How to avoid this instability?}

The first method was presented by the author in the 7th International MHD Conference of Warsaw [3, 4]. The method, after a previous calculation, was evidenced successfully in a shock tube MHD Faraday converter. The principle is very simple. When the gas (argon) whose temperature is $T_{\mathrm{g}}(4000 \mathrm{~K})$ enters the MHD channel, the applied transverse $B$-field $(2 \mathrm{~T})$ gives a value $R>8$, based on the electron-gas collision frequency $\nu_{\mathrm{en}}$ (weakly ionized gas). But the induced $V \times B$ electric field produces a non-thermal ionization, which grows fast enough

* www.mhdprospects.com/lambda/ 
to transform the plasma into a fully ionized gas (Coulomb collision dominated). As the electron-ion cross-section is $10^{4}$ larger than the electron-neutral cross-section, this pulls the local value of the Hall parameter below the critical value. Experiments with $T_{\mathrm{g}}=6000 \mathrm{~K}, T_{\mathrm{e}}=10000 \mathrm{~K}$ were achieved successfully in one bar pressure noble gas (pure argon or helium-argon mixtures), produced by a shock tube (velocity $=1900 \mathrm{~m} / \mathrm{s}$ ). The concept is illustrated in Fig. 2.

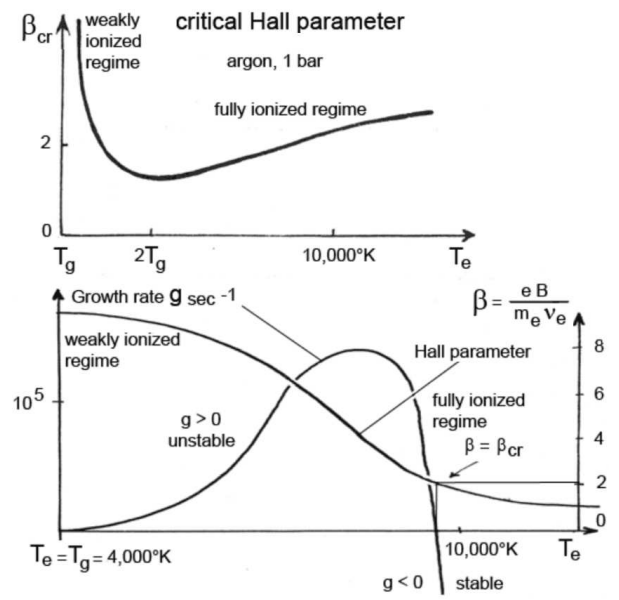

Fig. 2. Critical Hall parameter and growth rate of the instability in a non-equilibrium plasma.

Bottom: when entering the channel with a $2 \mathrm{~T}$ transverse magnetic field, the local value of the Hall parameter in the plasma $R>8$, higher than the critical value, so that the instability tends to grow. But the ionization extends, it modifies the collision frequency. When electron-heavy particles Coulomb-collisions dominate, the Hall parameter falls and the plasma becomes stable. Figure 3 is a picture showing the development of the ionization, from left to right, and the subsequent decrease of the instability. Bright spots, up and down: electrodes locations.

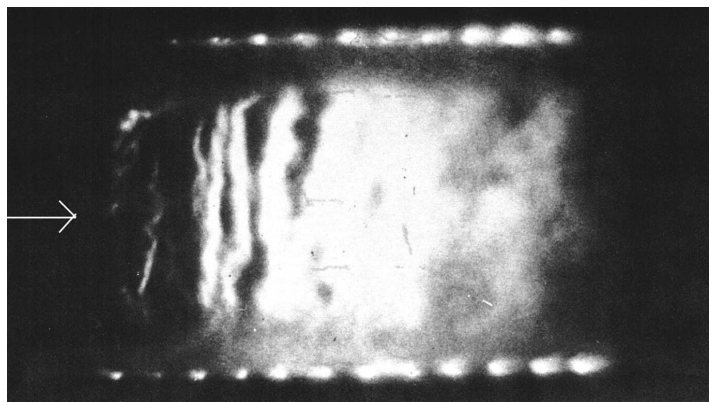

Fig. 3. The development of the ionization competes against the Velikhov instability and wins.

But it was not possible to go below $T_{\mathrm{g}}=4000 \mathrm{~K}$, so these experiments had no industrial application and interest.

\section{A better method: cancellation of the instability by magnetic confinement}

The electrical conductivity is

$$
\sigma=\sigma_{\mathrm{s}}\left[\begin{array}{cc}
\frac{1}{1+\beta^{2}} & \frac{-\beta}{1+\beta^{2}} \\
\frac{\beta}{1+\beta^{2}} & \frac{1}{1+\beta^{2}}
\end{array}\right],
$$

where $\sigma_{\mathrm{s}}$ is the scalar conductivity. Instead of suffering inhomogeneities, let us introduce a non-homogeneous $B$-field and shape it, tracing some alleys with weaker $B$ value, offering higher conductivity for the current. First, consider a linear channel as seen in Fig. 4. See Ref. [5] for details.

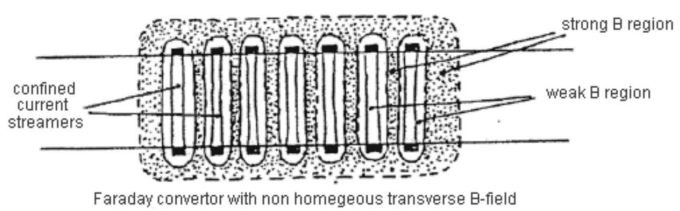

Fig. 4. Faraday MHD converter with non homogeneous magnetic fields.

We obtained thin linear current streamers in low pressure air experiments in the beginning of the eighties. The discharges experience a strong confinement effect, due to the non-homogeneous electrical conductivity. It increases the electron temperature, the ionization level. The transition to a fully ionized regime gives a favorable strong nonlinear response. The Velikhov instability cannot take place in such streamers. Moreover, this technique makes possible to control completely the electric discharge pattern by magnetic confinement. In other experiments, still in low pressure air (0.8 Torr) we used a 3-cathode disk accelerator with a central anode (Fig. 5).

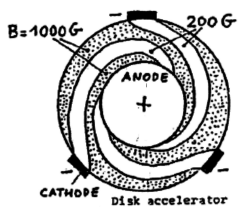

Fig. 5. Disk accelerator.

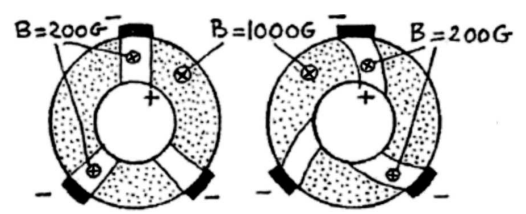

Fig. 6. Reverse current spiral due to magnetic confinement.

With a constant $B$-field (1000 Gs) the discharges created by a $1 \mathrm{kV}$ electric power supply were ugly, grooved by the Velikhov instability. According to the classical 
theory, the current refused to get a pitch angle higher than $45^{\circ}$. Introducing attenuated $B$-field spiral alleys, we got good looking thin spiral cords of plasma, corresponding to an apparent Hall parameter up to 5. Not only this magnetic confinement effect completely cancels the undesirable Velikhov instability, but it provides an efficient technique to control the streamers geometry. For example, this effect makes possible to reverse the whirling, is a disc-shaped machine as seen in Fig. 6.

\section{Conclusions}

If this method for cancelling the Velikhov instability and controlling the streamer geometry had been discovered in the sixties, it would perhaps have prevented various countries from giving up the international effort made to develop MHD generators with non-equilibrium ionization. Unfortunately, it was imagined more than ten years after all this research was abandoned. When I came to
Moscow in 1983, for the 8th International MHD Conference, I could meet my old good friend V. Golubev, E. Velikhov's coworker, who made this comment to me:

- You bring the cure. But the patient already died...

\section{References}

[1] E. Velikhov, in: Proc. 1st Int. Conf. on MHD Electrical Power Generation, Newcastle (England) 1962, p. 47.

[2] J.P. Petit, J. Valensi, Compt. Rend. Acad. Sci. Paris 269, (1969).

[3] J.P. Petit, J.P. Caressa, J. Valensi, in: Proc. Int. Symp. on MHD Electrical Power Generation, Warsaw (Poland) S.M. 107/123: 745 (1968).

[4] J.P. Petit, M. Billiotte, Compt. Rend. Acad. Sci. Paris 292, 158 (1981).

[5] J.P. Petit, in: Proc. 8th Int. Conf. on MHD Electrical Power Generation, Moscow (Russia) 1983. 